Pacific Journal of Mathematics

ON THE FINEST LEBESGUE TOPOLOGY ON THE SPACE OF 


\section{ON THE FINEST LEBESGUE TOPOLOGY ON THE SPACE OF ESSENTIALLY BOUNDED MEASURABLE FUNCTIONS}

\section{MARIAN NOWAK}

Let $(\Omega, \Sigma, \mu)$ be a $\sigma$-finite measure space and let $\mathscr{T}_{0}$ and $\mathscr{T}_{\infty}$ denote the usual metrizable topologies on $L^{0}$ and $L^{\infty}$, respectively. In this paper the space $L^{\infty}$ with the mixed topology $\gamma\left(\mathscr{T}_{\infty},\left.\mathscr{T}_{0}\right|_{L^{\infty}}\right)$ is examined. It is proved that $\gamma\left(\mathscr{T}_{\infty},\left.\mathscr{T}_{0}\right|_{L}\right)$ is the finest Lebesgue topology on $L^{\infty}$, and that it coincides with the Mackey topology $\tau\left(L^{\infty}, L^{1}\right)$.

1. Introduction. For notation and terminology concerning Riesz spaces and locally solid topologies we refer to [1].

Let $(\Omega, \Sigma, \mu)$ be a $\sigma$-finite measure space, and let $L^{0}$ denote the set of equivalence classes of all real valued $\mu$-measurable functions defined and finite a.e. on $\Omega$. Then $L^{0}$ is a super Dedekind complete Riesz space under the ordering $x \leq y$, whenever $x(t) \leq y(t)$ a.e. on $\Omega$. The Riesz $F$-norm

$$
\|x\|_{0}=\int_{\Omega}|x(t)|(1+|x(t)|)^{-1} f(t) d \mu \quad \text { for } x \in L^{0},
$$

where a function $f: \Omega \rightarrow(0, \infty)$ is $\mu$-measurable with $\int_{\Omega} f(t) d \mu=1$, determines a Lebesgue topology on $L^{0}$, which we will denote by $\mathscr{T}_{0}$ (see [7, I, §6], [1, Theorem 24.67]). This topology generates convergence in measure on the measurable subsets of $\Omega$ whose measure is finite. We will denote by $\mathscr{T}_{\infty}$ the topology on $L^{\infty}$ generated by the usual $B$-norm

$$
\|x\|_{\infty}=\underset{t \in \Omega}{\operatorname{ess} \sup }|x(t)| .
$$

Moreover, we denote by $\sigma\left(L^{\infty}, L^{1}\right), \tau\left(L^{\infty}, L^{1}\right)$ and $\beta\left(L^{\infty}, L^{1}\right)$ the weak, Mackey and strong topologies on $L^{\infty}$ respectively, with respect to the dual pair $\left(L^{\infty}, L^{1},\langle\rangle,\right)$, where

$$
\langle x, y\rangle=\int_{\Omega} x(t) y(t) d \mu \quad \text { for } x \in L^{\infty}, y \in L^{1} .
$$

In this paper we shall examine the space $L^{\infty}$ with the mixed topology $\gamma\left(\mathscr{T}_{\infty},\left.\mathscr{T}_{0}\right|_{L^{\infty}}\right)$. This topology is defined as follows. Take a sequence 
$\left(\varepsilon_{n}\right)$ of positive numbers, a number $r>0$ and let

$$
W\left(\left(\varepsilon_{n}\right), r\right)=\bigcup_{N=1}^{\infty}\left(\sum_{n=1}^{N} V\left(\varepsilon_{n}\right) \cap n B(r)\right),
$$

where $B(r)=\left\{x \in L^{\infty}:\|x\|_{\infty} \leq r\right.$ and $V\left(\varepsilon_{n}\right)=\left\{x \in L^{\infty}:\|x\|_{0} \leq \varepsilon_{n}\right\}$. Then the family of all such $W\left(\left(\varepsilon_{n}\right), r\right)$ forms a base of neighbourhoods of zero for $\gamma\left(\mathscr{T}_{\infty},\left.\mathscr{T}_{0}\right|_{L^{\infty}}\right)$ (see [11, p. 49]). In view of [11, Theorem 2.2.2] $\gamma\left(\mathscr{T}_{\infty},\left.\mathscr{T}_{0}\right|_{L^{\infty}}\right)$ is the finest linear topology on $L^{\infty}$ which agrees with $\left.\mathscr{T}_{0}\right|_{L^{\infty}}$ on $\|\cdot\|$-bounded sets. Henceforth, we will write briefly $\gamma$ instead of $\gamma\left(\mathscr{T}_{\infty},\left.\mathscr{T}_{0}\right|_{L^{\infty}}\right)$.

The space of bounded sequences $l^{\infty}$ with the mixed topology $\gamma$ has been investigated in [4], where among other things, the results from Theorems 5, 6 and 8 below are obtained. The mixed topology $\gamma$ on $l^{\infty}$ is the same as the strict topology $\beta$ [3] on $C(S)$, where $S=N=$ the set of all natural numbers.

2. The mixed topology $\gamma$ on $L^{\infty}$. It is well known that the norm topology $\mathscr{T}_{\infty}$ on $L^{\infty}$ satisfies both the Fatou property and the Levi property (see $[7, I V, \S 3]$ and $[7, X, \S 4])$, and that $\mathscr{T}_{\infty}$ does not satisfy the Lebesgue property if $\Omega$ does not consist of only finite number of atoms (see [7, IV, §3]). We shall show that the mixed topology $\gamma$ is the finest Hausdorff Lebesgue topology on $L^{\infty}$. We start by giving some characterization of sequential convergence in $\left(L^{\infty}, \gamma\right)$.

THEOREM 1. For a sequence $\left(x_{n}\right)$ in $L^{\infty}, x_{n} \rightarrow 0$ for $\gamma$ if and only if $\left\|x_{n}\right\|_{0} \rightarrow 0$ and $\left\|x_{n}\right\|_{\infty}<M$ for some $M>0$ and all $n=1,2, \ldots$

Proof. Since the balls $B(r)=\left\{x \in L^{\infty}:\|x\|_{\infty} \leq r\right\}, r>0$ are closed in $\mathscr{T}_{0}$ (see [7, IV, §3, Lemma 5]) the result follows from [11, Theorem 2.3.1].

We now are able to prove the basic property of $\gamma$.

THEOREM 2. The mixed topology $\gamma$ is the finest Hausdorff Lebesgue topology on $L^{\infty}$.

Proof. Using [1, Theorem 1.2] it is easy to show that $\gamma$ is a locally solid topology. In order to show that $\gamma$ is a Lebesgue topology, let us assume that $x_{\alpha} \downarrow 0$ holds in $L^{\infty}$ and let $\left(\varepsilon_{n}\right)$ be a sequence of positive numbers and $r>0$. Then there exists an increasing sequence of indices $\left\{\alpha_{n}\right\} \subset\{\alpha\}$ such that $x_{\alpha_{n}} \downarrow 0$ holds in $L^{\infty}$, because 
$L^{\infty}$ has the countable sup property (see [9, Proposition 5.20]). Since $\mathscr{T}_{0}$ is a Lebesgue topology, we have $x_{\alpha_{n}} \rightarrow 0$ for $\gamma$ by Theorem 1 . Then there exists a natural number $n_{0}$ such that $x_{\alpha_{n_{0}}} \in W\left(\left(\varepsilon_{n}\right), \tau\right)$, so $x_{\alpha} \in W\left(\left(\varepsilon_{n}\right), r\right)$ for $\alpha \geq \alpha_{n_{0}}$, and hence $x_{\alpha} \rightarrow 0$ for $\gamma$. Now let $\xi$ be a Hausdorff Lebesgue topology on $L^{\infty}$. Then by [1, Theorem 12.9] we have $\xi_{[-x, x]}=\left.\mathscr{T}_{0}\right|_{[-x, x]}$ for every $0<x \in L^{\infty}$. Hence, by [11, Theorem 2.2.2] the inclusion $\xi \subset \gamma$ holds, and thus the proof is finished.

REMARK. It is known that $L^{\infty}$ has no minimal topology, if the measure $\mu$ is atomless [2].

We now consider the problem of separableness of the space $\left(L^{\infty}, \gamma\right)$. First, we recall some definition. Let $\sim$ be the following equivalence relation in $\Sigma: A \sim B$ if and only if $\mu(A \dot{-} B)=0(\dot{-}$ denotes the symmetric difference). Denote by $\Sigma / \sim$ the set of equivalence classes and by $[A]$ the equivalence class of $A$. Then on $\Sigma / \sim$ one can define a metric function $\rho([A],[B])=\left\|\chi_{A}-\chi_{B}\right\|_{0} .\left(\chi_{A}\right.$ denotes the characteristic function of the set $A$.) The measure $\mu$ is said to be separable if the metric space $(\Sigma / \sim, \rho)$ is separable (see $[7, I, \S 6])$.

THEOREM 3. The space $\left(L^{\infty}, \gamma\right)$ is separable if and only if the measure $\mu$ is separable.

Proof. Assume that the space $\left(L^{\infty}, \gamma\right)$ is separable and let $0<x \in$ $L^{0}$. Let $x_{n}=x \wedge n e$, where $e$ denotes the constant function one. Then $0 \leq x_{n} \uparrow x$ holds in $L^{0}$, so $x_{n} \rightarrow x$ for $\mathscr{T}_{0}$. Thus $L^{\infty}$ is dense in $\left(L^{0}, \mathscr{T}_{0}\right)$, hence $\left(L^{0}, \mathscr{T}_{0}\right)$ is separable by hypothesis $[7,1, \S 6]$. By [7, I, $\S 6$, Theorem 16] the measure $\mu$ is separable.

Next, assume that the measure $\mu$ is separable. Let

$$
\begin{aligned}
\mathscr{P}=\left\{\sum_{k=1}^{m} c_{k} \chi_{A_{k}}: A_{k} \in \Sigma, \mu\left(A_{k}\right)<\infty,\right. & \\
& \left.A_{k_{1}} \cap A_{k_{2}}=\varnothing \text { for } k_{1} \neq k_{2}, c_{k} \in \mathbf{R}, m \in \mathbf{N}\right\}
\end{aligned}
$$

where $\mathbf{R}$ denotes the set of real numbers. Then $\mathscr{P} \subset L^{\infty}$ and using Theorem 1, by usual argument one can show that the set $\mathscr{P}$ is dense in $\left(L^{\infty}, \gamma\right)$. Let $\Sigma_{0}$ be a countable subset of $\Sigma / \sim$, which is dense in $(\Sigma / \sim, \rho)$. Let $\mathscr{P}_{0}=\left\{\sum_{k=1}^{m} r_{k} \chi_{A_{k}} \in \mathscr{P}:\left[A_{k}\right] \in \Sigma_{0}, r_{k} \in \mathbf{Q}\right\}$, where $\mathbf{Q}$ denotes the set of rational numbers. Let $0 \leq x=\sum_{k=1}^{m} c_{k} \chi_{A_{k}} \in \mathscr{P}$. Then, by hypothesis, for every $k=1, \ldots, m$ there exist a sequence 
$\left(\left[A_{k}^{n}\right]\right)$ in $\Sigma_{0}$ and a sequence $\left(r_{k}^{n}\right)$ of positive rational numbers such that $\left\|\chi_{A_{k}^{n}}-\chi_{A_{k}}\right\|_{0} \rightarrow 0$ as $n \rightarrow \infty$ and $0 \leq r_{k}^{n} \uparrow_{n} c_{k}$ for $k=1, \ldots, m$. Putting $x_{n}=\sum_{k=1}^{m} r_{k}^{n} \chi_{A_{k}^{n}}$ for $n=1,2, \ldots$, we have $\left\|x_{n}-x\right\|_{0} \rightarrow 0$ and $\left|x_{n}(t)\right| \leq \max _{1 \leq k \leq m} c_{k}$ a.e. on $\Omega$. Thus, by Theorem $1, x_{n} \rightarrow x$ for $\gamma$. It follows that the set $\mathscr{P}_{0}$ is dense in $(\mathscr{P}, \gamma \mid \mathscr{P})$, so $\mathscr{P}_{0}$ is dense also in $\left(L^{\infty}, \gamma\right)$. Thus the space $\left(L^{\infty}, \gamma\right)$ is separable, because the set $\mathscr{P}_{0}$ is countable.

The next theorem describes the topological dual of $\left(L^{\infty}, \gamma\right)$.

THEOREM 4. For a linear functional $f$ on $L^{\infty}$ the following statements are equivalent:

(i) $f$ is continuous for $\gamma$.

(ii) $f$ is sequentially continuous for $\gamma$.

(iii) There exists a unique $y \in L^{1}$ such that

$$
f(x)=\int_{\Omega} x(t) y(t) d \mu \quad \text { for } x \in L^{\infty} .
$$

Proof. (i) $\Leftrightarrow$ (ii) It follows from [11, Theorem 2.6.1].

(ii) $\Leftrightarrow$ (iii) By Theorem 1 , the functional $f$ is sequentially continuous for $\gamma$ if and only if it is sequentially order star-continuous, and if and only if it is sequentially order continuous (cf. [6, VII, §2]). Thus, in view of [7, VI, $\S 2$, Theorem 1] the proof is finished.

As an application of Theorems 2 and 4 we get the following important property of $\gamma$.

THEOREM 5. The mixed topology $\gamma$ on $L^{\infty}$ is a Mackey topology, i.e., $\gamma=\tau\left(L^{\infty}, L^{1}\right)$.

Proof. Since the Mackey topology $\tau\left(L^{\infty}, L^{1}\right)$ is a Lebesgue topology (see [1, Ex. 4, p. 163] and [1, Theorem 9.1]), by Theorem 2 we have $\tau\left(L^{\infty}, L^{1}\right) \subset \gamma$. According to Theorem 4 , it suffices to show that $\gamma$ is a locally convex topology. Indeed, let us put $x_{n}(t)=n$ for $t \in \Omega$ and $n=1,2, \ldots$ Let $\mathscr{T}_{I}$ be the generalized inductive limit topology of $\left(L^{\infty}, \tau\left(L^{\infty}, L^{1}\right), j_{n},\left[-x_{n}, x_{n}\right]\right)\left(\right.$ see $\left[5\right.$, p. 2]), i.e., $\mathscr{T}_{I}$ is the finest of all locally convex topologies $\xi$ on $L^{\infty}$ under which the inclusion maps

$$
j_{n}:\left(\left[-x_{n}, x_{n}\right],\left.\tau\left(L^{\infty}, L^{1}\right)\right|_{\left[-x_{n}, x_{n}\right]}\right) \rightarrow\left(L^{\infty}, \xi\right)
$$

are continuous for $n=1,2, \ldots$ By [5, Proposition 5] $\mathscr{T}_{I}$ is also the finest of all linear topologies $\xi$ on $L^{\infty}$ under which each of the maps $j_{n}$ 
is continuous. Since $\gamma$ and $\tau\left(L^{\infty}, L^{1}\right)$ are Hausdorff Lebesgue topologies, by [1, Theorem 12.9] we have

$$
\gamma_{\left[-x_{n}, x_{n}\right]}=\left.\tau\left(L^{\infty}, L^{1}\right)\right|_{\left[-x_{n}, x_{n}\right]} \quad \text { for } n=1,2, \ldots
$$

Thus $\gamma \subset \mathscr{T}_{I}$. On the other hand, since

$$
\left.\left.\mathscr{T}_{1}\right|_{\left[-x_{n}, x_{n}\right]} \subset \tau\left(L^{\infty}, L^{1}\right)\right|_{\left[-x_{n}, x_{n}\right]}=\left.\mathscr{T}_{0}\right|_{\left[-x_{n}, x_{n}\right]} \quad \text { for } n=1,2, \ldots,
$$

by [11, Theorem 2.2.2] we get $\mathscr{T}_{I} \subset \gamma$. Thus $\mathscr{T}_{I}=\gamma$; hence $\gamma$ is locally convex. Therefore, we have $\gamma \subset \tau\left(L^{\infty}, L^{1}\right)$. Thus the proof is finished.

For a linear topology $\mathscr{T}$ on $L^{\infty}$, we will denote by $\operatorname{Bd}(\mathscr{T})$ the collection of all $\mathscr{T}$-bounded subsets of $L^{\infty}$.

Additional properties of $\gamma$ are included in the next theorem.

THEOREM 6. The space $L^{\infty}$ endowed with $\gamma$ is complete.

Proof. Since $\gamma$ is a Lebesgue topology, in view of [1, Theorem 13.9] it suffices to show that $\gamma$ is a Levi topology. $\operatorname{But} \operatorname{Bd}(\gamma)=\operatorname{Bd}\left(\mathscr{T}_{\infty}\right)[11$, Theorem 2.4.1], so $\gamma$ is a Levi topology, because we know that $\mathscr{T}_{\infty}$ is a Levi topology.

COROllary 7. The mixed topology $\gamma$ is not metrizable.

Locally convex Hausdorff space $(X, \xi)$ is called sequentially barreled if every $\sigma\left(X^{*}, X\right)$-convergent to zero sequence in the topological dual $X^{*}=(X, \xi)^{*}$ is equicontinuous $[\mathbf{1 0}]$.

THEOREM 8. The space $\left(L^{\infty}, \gamma\right)$ is sequentially barreled.

Proof. Combining Theorem 4 and Theorem 5, we have $\gamma=$ $\tau\left(L^{\infty},\left(L^{\infty}, \gamma\right)^{+}\right)$, where $\left(L^{\infty}, \gamma\right)^{+}$denotes the sequential topological dual of $\left(L^{\infty}, \gamma\right)$. Since the space $\left(L^{\infty}, \gamma\right)$ is complete, according to [10, Proposition 4.3] the space $\left(L^{\infty}, \gamma\right)$ is sequentially barreled.

Since $L^{\infty}$ is the norm dual of $L^{1}$ we have $\beta\left(L^{\infty}, L^{1}\right)=\mathscr{T}_{\infty}$. Therefore, according to Theorem 4 and Corollary 7 we obtain that the space $\left(L^{\infty}, \gamma\right)$ is not barreled.

Additional characterizations of sequential convergence in $\left(L^{\infty}, \gamma\right)$ are included in the next theorem. 
THEOREM 9. For a sequence $\left(x_{n}\right)$ in $L^{\infty}$ the following statements are equivalent:

(i) $x_{n} \rightarrow 0$ for $\gamma$.

(ii) $x_{n} \rightarrow 0$ for the absolutely weak topology $|\sigma|\left(L^{\infty}, L^{1}\right)$.

(iii) $\int_{\Omega}\left|x_{n}(t) y(t)\right| d \mu \rightarrow 0$ for every $y \in L^{1}$.

Proof. (i) $\Leftrightarrow$ (ii) Since $|\sigma|\left(L^{\infty}, L^{1}\right) \subset \tau\left(L^{\infty}, L^{1}\right)$ (see [1, Theorem 6.7], assume that $x_{n} \rightarrow 0$ for $|\sigma|\left(L^{\infty}, L^{1}\right)$. By [1, Theorem 12.9] we have $\left.|\sigma|\left(L^{\infty}, L^{1}\right)\right|_{[-x, x]}=\left.\mathscr{T}_{0}\right|_{[-x, x]}$ for every $0<x \in L^{\infty}$, because $|\sigma|\left(L, L^{1}\right)$ is a Hausdorff Lebesgue topology. Since the set $\left\{x_{n}\right\}$ is $\sigma\left(L, L^{1}\right)$-bounded and $\operatorname{Bd}\left(\sigma\left(L^{\infty}, L^{1}\right)\right)=\operatorname{Bd}\left(\tau\left(L^{\infty}, L^{1}\right)\right)=\operatorname{Bd}\left(\tau_{\infty}\right)$ we obtain that $\left\{x_{n}\right\} \subset[-x, x]$ for some $0<x \in L^{\infty}$. Thus $\left\|x_{n}\right\|_{0} \rightarrow 0$, and in view of Theorem 1 we have $x_{n} \rightarrow 0$ for $\gamma$.

(ii) $\Leftrightarrow$ (iii) Obvious.

The next theorem gives criteria for the compactness of sets in $\left(L^{\infty}, \gamma\right)$.

THeOREM 10. For a subset $Z$ of $L^{\infty}$ the following statements are equivalent:

(i) $Z$ is relatively compact for $\mathscr{T}_{0}$ and $\|x\|_{\infty}<M$ for some $M>0$ and every $x \in Z$.

(ii) $Z$ is relatively compact for $\gamma$.

(iii) $Z$ is relatively compact for $|\sigma|\left(L^{\infty}, L^{1}\right)$.

Proof. (i) $\Leftrightarrow$ (ii) Obvious, because we know that $\operatorname{Bd}\left(\mathscr{T}_{\infty}\right)=\operatorname{Bd}(\gamma)$ and the topologies $\gamma$ and $\mathscr{T}_{0}$ coincide on order intervals of $L^{\infty}$.

(ii) $\Rightarrow$ (iii) Obvious, because $|\sigma|\left(L^{\infty}, L^{1}\right) \subset \gamma$.

(iii) $\Rightarrow$ (ii) Combining [8, I, $\S 3$, Lemma 11] and Theorem $9, Z$ is relatively compact for $\gamma$.

\section{REFERENCES}

[1] C. D. Aliprantis and O. Burkinshaw, Locally Solid Riesz Spaces, Academic Press, New York, 1978.

[2] Minimal topologies and $L_{p}$-spaces, Illinois J. Math., 24 (1980), 164172.

[3] R. C. Buck, Bounded continuous functions on a locally compact space, Michigan Math. J., 5 (1958), 95-104. 
[4] A. K. Chilana, The space of bounded sequences with mixed topology, Pacific J. Math., 48 (1973), 29-33.

[5] D. J. H. Garling, A generalized form of inductive-limit topology for vector spaces, Proc. London Math. Soc., 14 (1964), 1-28.

[6] L. V. Kantorovich, B. Z. Vulikh and A. Pinsker, Functional Analysis on Semiordered Spaces, Moscow-Leningrad, 1950 (Russian).

[7] L. V. Kantorovich and G. P. Akilov, Functional Analysis, Moscow, 1984 (Russian).

[8] W. A. Luxemburg, Banach Function Spaces, Delft 1955.

[9] A. Peressini, Ordered Topological Vector Spaces, Harper and Row, London, 1967.

[10] J. H. Webb, Sequential convergence in locally convex spaces, Proc. Camb. Phil. Soc., 64 (1968), 341-364.

[11] A. Wiweger, Linear spaces with mixed topology, Studia Math., 20 (1961), 47-68.

Received November 15, 1987.
A. MickiewiCZ UNIVERSITY
MatejKI 48/49
60-769 Poznan, Poland 



\section{PACIFIC JOURNAL OF MATHEMATICS EDITORS}

\author{
V. S. VARADARAJAN \\ (Managing Editor) \\ University of California \\ Los Angeles, CA 90024-1555-05 \\ Herbert Clemens \\ University of Utah \\ Salt Lake City, UT 84112 \\ THOMAS ENRIGHT \\ University of California, San Diego \\ La Jolla, CA 92093
}

R. FINN

Stanford University

Stanford, CA 94305

HeRMANN FlaschKa

University of Arizona

Tucson, AZ 85721

VAUGHaN F. R. Jones

University of California

Berkeley, CA 94720

STEVEN KeRCKHOFF

Stanford University

Stanford, CA 94305

\author{
ROBION KIRBY \\ University of California \\ Berkeley, CA 94720 \\ C. C. MOORE \\ University of California \\ Berkeley, CA 94720 \\ HAROLD STARK \\ University of California, San Diego \\ La Jolla, CA 92093
}

\section{ASSOCIATE EDITORS}
R. ARENS
E. F. BECKENBACH
B. H. NEUMANN
F. WOLF
K. YoshidA (1906-1982)

\section{SUPPORTING INSTITUTIONS}

UNIVERSITY OF ARIZONA

UNIVERSITY OF BRITISH COLUMBIA

CALIFORNIA INSTITUTE OF TECHNOLOGY

UNIVERSITY OF CALIFORNIA

MONTANA STATE UNIVERSITY

UNIVERSITY OF NEVADA, RENO

NEW MEXICO STATE UNIVERSITY

OREGON STATE UNIVERSITY
UNIVERSITY OF OREGON

UNIVERSITY OF SOUTHERN CALIFORNIA

STANFORD UNIVERSITY

UNIVERSITY OF HAWAII

UNIVERSITY OF TOKYO

UNIVERSITY OF UTAH

WASHINGTON STATE UNIVERSITY

UNIVERSITY OF WASHINGTON 


\section{Pacific Journal of Mathematics}

Vol. 140, No. $1 \quad$ September, 1989

Michel Brestovski, Algebraic independence of solutions of differential

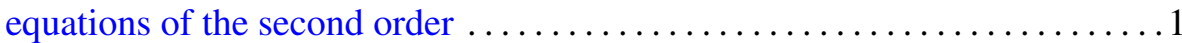

Bohumil Cenkl, Cohomology operations from higher products in the de

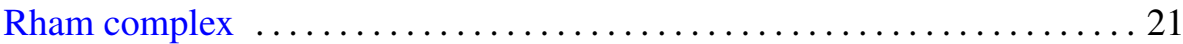

Gustavo Corach and Daniel Suarez, Generalized rational convexity in

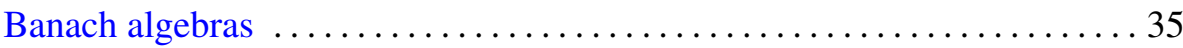

Keresztély Corrádi and Sándor Szabó, A new proof of Rédei’s theorem . . . 53

Steven R. Costenoble and Stefan Waner, Equivariant orientations and

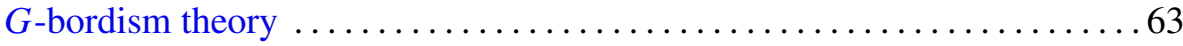

Angel Granja, Apéry basis and polar invariants of plane curve

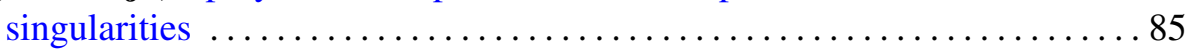

Young Soo Jo, Isometries of tridiagonal algebras .................. 97

Ronald Leslie Lipsman, Harmonic analysis on exponential solvable homogeneous spaces: the algebraic or symmetric cases $\ldots \ldots \ldots \ldots \ldots 117$

Erich Miersemann, On the behaviour of capillaries at a corner 149

Marian Nowak, On the finest Lebesgue topology on the space of essentially

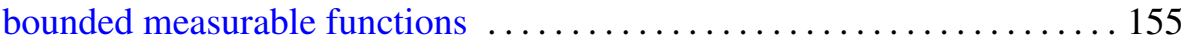

Pascal J. Thomas, Hardy interpolating sequences of hyperplanes ........ 163

H. Bevan Thompson, Differentiability properties of subfunctions for second order ordinary differential equations 